\title{
Organization and Expression of the Pyruvate Dehydrogenase Complex Genes of Escherichia coli K12
}

\author{
By JOHN R. GUEST, * STEWART T. COLE† AND \\ KANDIAH JEYASEELANł \\ Department of Microbiology, University of Sheffield, Sheffield S10 2TN, U.K.
}

(Received 6 May 1981)

The organization and expression of the pyruvate dehydrogenase complex genes, aceE (E1), aceF (E2) and lpd (E3), were investigated using a series of $\lambda$ transducing phages carrying different segments of the nadC-aroP-aceE-aceF-lpd region of the chromosome of Escherichia coli. The polypeptides synthesized following the infection of u.v.-irradiated lysogenic and non-lysogenic hosts were analysed by sodium dodecyl sulphate-polyacrylamide gel electrophoresis and autoradiography.

The ace $E$ and $l p d$ gene products were readily identified by their sizes, $M_{\mathrm{r}} 100000$ (pyruvate dehydrogenase, E1 component) and 56500 (lipoamide dehydrogenase, E3 component), but considerable heterogeneity was detected for the aceF gene product (acetyltransferase, E2 component). The main E2 polypeptides, $M_{\mathrm{r}} 80000$ and 83000 , were accompanied by a family of minor polypeptides, $M_{\mathrm{r}} 86000,89000$ and 91000 , but no precursor-product relationships were apparent. A very large polypeptide, $M_{\mathrm{r}} 190000$, was also detected and found to contain the $\mathrm{E} 1$ component but in uncertain combination. In addition, the product of a gene in the nadC-aroP region was detected as a polypeptide with $M_{\mathrm{r}} 36500$.

The existence of a single promoter for the $a c e E$ and $a c e F$ genes and a separate promoter for the lpd gene was confirmed. The lpd gene was shown to be transcribed with the same polarity as the ace genes (clockwise with respect to the $E$. coli linkage map). The relative rates of expression of the three genes from the bacterial promoters were estimated as $0.94: 1 \cdot 0: 1.4-$ $2 \cdot 3$ (E1:E2:E3) on a molar basis. The excess production of lipoamide dehydrogenase components (E3) indicates that the $l p d$ promoter functions independently, at least in supplying components for the 2-oxoglutarate dehydrogenase complex. When expressed from $\mathrm{P}_{\mathrm{L}}$, the powerful phage promoter, the ace and lpd gene products accounted for the greater part of the newly synthesized protein.

\section{INTRODUCTION}

The pyruvate dehydrogenase complex of Escherichia coli is a multienzyme complex catalysing the overall reaction:

$$
\text { Pyruvate }+\mathrm{NAD}^{+}+\mathrm{CoASH} \rightarrow \text { Acetyl-SCoA }+\mathrm{NADH}^{+}+\mathrm{H}^{+}+\mathrm{CO}_{2}
$$

It contains multiple copies of three enzymic components: E1, pyruvate dehydrogenase (EC 1.2.4.1); E2, dihydrolipoamide acetyltransferase (EC 2.3.1.12); and E3, lipoamide dehydrogenase (EC 1.6.4.3). Recent estimates of the molecular weights $\left(M_{\mathrm{r}}\right)$ of the corresponding polypeptides are 100000 for E1, 83000 and 89000 for E2, and 56500 for E3 (Vogel, 1977; Gebhardt et al., 1978), although there is evidence that the E2 polypeptide may be much smaller $\left(M_{\mathrm{r}} 64500\right.$; Bleile et al., 1979). The complex has a structural core comprised

\footnotetext{
† Present address: Max-Planck-Institut für Biologie, Correnstrasse 38, 7400 Tübingen 1, West Germany. $\ddagger$ Present address: Department of Botany, University of Jaffna. Sri Lanka.
} 
of E2 components surrounded by independently bound E1 and E3 components. However, the size and polypeptide chain stoichiometry of the native complex, if constant, are still uncertain. A recent study has indicated that the major fraction of the isolated complex has a molecular weight of $6 \times 10^{6}$, corresponding to a core of $24 \mathrm{E} 2$ chains, and a disproportionate chain ratio (E1 :E2:E3) of 1.6:1 0:0.8 (Danson et al., 1979). There is also evidence that the structure of the complex is not unique and that it exhibits a limited degree of heterogeneity (Schmitt \& Cohen, 1980).

The dehydrogenase (E1) and acetyltransferase (E2) structural genes (aceE and aceF) constitute an operon with ace $\overrightarrow{E F}$ polarity, which is very close to the nadC (quinolinate phosphoribosyltransferase) and aroP (general aromatic amino acid permease) genes at $2.6 \mathrm{~min}$ on the $E$. coli linkage map (Guest, 1978). The lipoamide dehydrogenase (E3) structural gene (lpd) is linked to the $a c e F$ gene thus: nadC-aroP-aceE-aceF-lpd. The specific components of the analogous 2-oxoglutarate dehydrogenase complex are encoded by the sucA and $s u c B$ genes of the $s u c$ operon, which is situated at $16.4 \mathrm{~min}$ and has the same functional polarity, suc $\overrightarrow{A B}$ (Guest, 1978). The E3 components of the 2-oxoglutarate dehydrogenase complex are encoded by the identical aceF-linked lpd gene. This poses the interesting problem of how the expression of the single lpd gene is geared to the expression of the separately regulated ace and suc operons. Indirect evidence from biochemical studies with polar and deletion mutants supports the view that the lpd gene can be expressed independently of the ace and suc genes (Creaghan \& Guest, 1972; Langley \& Guest, 1978).

Recently, recombinant $\lambda$ phages containing different segments of the nadC to lpd region have been constructed (Guest \& Stephens, 1980) and a physical map of the corresponding chromosomal region has been deduced by restriction analysis. Furthermore, studies with dilysogens have proved that the cloned lpd gene can be transcribed from its own promoter in the absence of a functional ace operon and when the major $\lambda$ promoters are repressed. The polarity of lpd expression relative to the polarity of the ace operon was not established. In the present work the technique of analysing phage-specified proteins by labelling u.v.-irradiated cells has been used with a variety of artificially constructed transducing phages, $(a)$ to identify the products expressed in vivo from genes in the nadC to lpd region, $(b)$ to establish the corresponding polarities of gene expression relative to the phage and bacterial genomes, particularly for the lpd gene relative to the ace operon, and $(c)$ for further correlation of segments of the physical map with the corresponding genetic and biochemical functions.

\section{METHODS}

Phages and bacteria. The phages were almost all derived from one EcoRI vector. NM781 (srI $11-3, c I 857 \mathrm{nin} 5$, containing a replaceable $E$. coli supE fragment between $s r I \lambda 1$ and $s r I \lambda 3$ : Murray et al., 1977). They were originally isolated from artificially constructed hybrid phage pools of Dr N. E. Murray and characterized by Guest \& Stephens (1980). Some properties of these phages and their derivatives are shown in Fig. 1. Phages G78N $(\lambda n a d C)$. G82L $(\lambda l p d)$ and G83L ( $\lambda l p d)$ were selected for their ability to transduce the nadC and $l p d$ genes. They contain EcoRI fragments of 10.1 and 8.2 kilobase pairs $(\mathrm{kb})$ that are adjacent in the bacterial chromosome. The $8.2 \mathrm{~kb}$ fragment is inserted with opposite polarity in the two $\lambda l p d$ phages, G82L and G83L. Phage G78N contains a functional aroP gene and the EcoRI target shared by the $\lambda$ nadC and $\lambda l p d$ phages lies within the aceE gene. Phages with extended transducing activities were $\mathrm{G} 90$ ( $\lambda$ nadC-lpd: from $\mathrm{G} 78 \mathrm{~N}$ ) and $\mathrm{G} 93$ ( $\lambda l p d-a c e E$ : from G83L). Both contain functional ace operons. which are oppositely polarized relative to the phage genome, but only G90 contains the $a r o P$ gene. Another phage. G105 ( $\lambda l p d)$. was kindly provided by P. E. Stephens. It was constructed by inserting the $5.4 \mathrm{~kb}$ HindIII plus EcoRI double-digestion product between $s r I \lambda I / 2$ and $s h n \lambda 3$ of the vector phage NM540 $\left(\operatorname{sr} I \lambda 1-2_{\Delta} \operatorname{shn} \lambda 3^{+} a t t^{+} i m m^{21} \operatorname{nin} 5 \operatorname{shn} \lambda 6^{\circ}\right)$. The bacterial fragment was inserted with the same polarity as it occurs in G83L and G93.

Escherichia coli strain $\mathrm{S} 159$ ( sup $^{\circ}$ gal uvrA rpsL) and its lysogenic derivative. $\mathrm{S} 159\left(\lambda \mathrm{imm}^{\lambda} \mathrm{ind}^{-}\right)$, were used as u.v.-irradiated hosts for infection and pulse-labelling. Strain C600 ( $\mathrm{thr}$ leu thi supE tonA) was used for routine phage assays. The methods used for preparation and assay of high titre phage lysates have been described previously (Guest \& Stephens. 1980). 

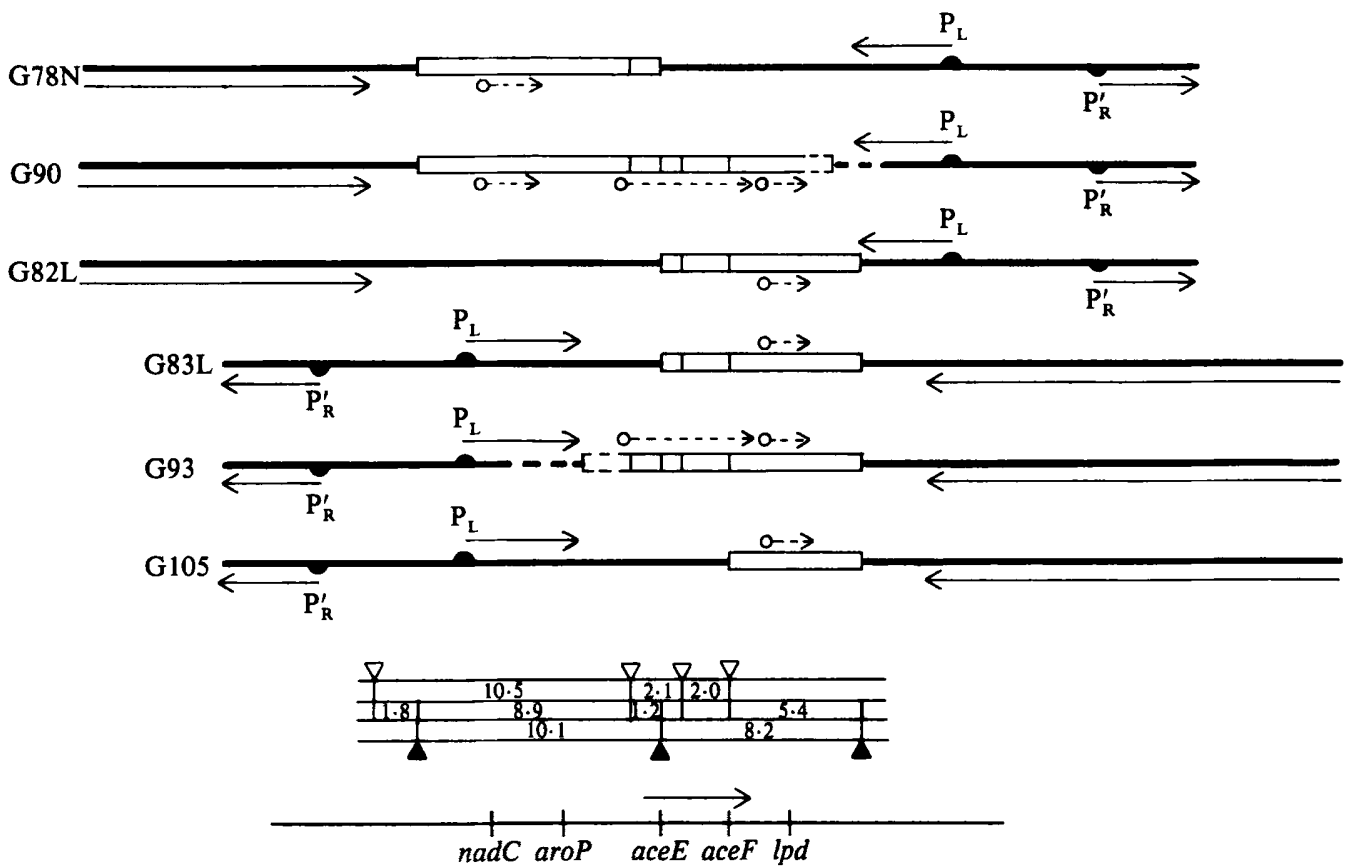

Fig. 1. The genomes of the $\lambda$ nadC and $\lambda l p d$ transducing phages and the phages with extended transducing activities ( $\lambda$ nadC-lpd, $\lambda l p d-a c e E$ ) of Guest \& Stephens (1980) are represented diagrammatically together with the physical and genetic maps of the corresponding segments of bacterial DNA. Phage G90 ( $\lambda$ nadC-lpd) was derived from G78N ( $\left.\lambda_{n a d C}\right)$ and the cloned DNA is inserted with the same polarity as in G82L ( $\lambda l p d)$. Phage G93 ( $\lambda l p d-a c e E$ ) was derived from G83L $(\lambda l p d)$ and in these phages, and G105 $(\lambda l p d)$, the bacterial DNA is inserted with the alternate polarity relative to the phage genome. The positions of the bacterial genes cloned in transducing phages are aligned with the bacterial linkage map $(2.5$ to $2.8 \mathrm{~min})$ and the physical map (where necessary, the vector arms have been reversed). The polarity of ace gene expression is denoted by the arrow above the genetic map. The dashed arrows indicate the polarities of expression of genes defined during the course of this work. Vector DNA is indicated by thick single lines and bacterial DNA by double lines. The broken lines denote regions where transition from bacterial to phage DNA is uncertain. The phage promoters, $P_{L}$ (early) and $P_{R}^{\prime}$ (late), are indicated by the - symbols on the phage DNA with arrows showing the direction of transcription. The restriction targets are denoted by the symbols $\Delta$ for $E c o$ RI and $\nabla$ for HindIII. The sizes of the corresponding restriction fragments are indicated in kilobase pairs (kb).

Analysis of polypeptides formed after u.v.-irradiation and phage infection. Cultures of $E$. coli S159 (or its $\lambda \mathrm{imm}^{\lambda}$ lysogen) were grown to a density of $10^{9}$ bacteria $\mathrm{ml}^{-1}$ in minimal medium (M9 salts: Miller, 1972) with glucose $(1 \%, \mathrm{w} / \mathrm{v})$ at $37^{\circ} \mathrm{C}$. They were resuspended at $4 \times 10^{\circ}$ bacteria $\mathrm{ml}^{-1}$ in fresh medium containing $\mathrm{MgSO}_{4}(20 \mathrm{~mm})$ and irradiated with u.v. light $\left(900 \mathrm{~J} \mathrm{~m}^{-2}\right)$. Samples $(100 \mu \mathrm{l})$ plus fresh medium $(400 \mu \mathrm{l})$ were infected at a multiplicity of $5\left(5 \mu \mathrm{l}\right.$ of $4 \times 10^{11}$ plaque-forming units $\left.\mathrm{ml}^{-1}\right)$ and pulse-labelled with $\mathrm{L}-\left[{ }^{35} \mathrm{~S}\right]$ methionine [final concentration 80-125 $\mu \mathrm{Ci} \mathrm{ml}^{-1}\left(2.96-4.63 \mathrm{MBq} \mathrm{m} l^{-1}\right)$; Amershaml for periods of 3-35 min at $37^{\circ} \mathrm{C}$. After incorporation, L-methionine was added to a concentration of $120 \mu \mathrm{g} \mathrm{m}^{-1}$ and the pelleted cells were washed in cold saline. The samples were prepared for sodium dodecyl sulphate (SDS)-polyacrylamide gel electrophoresis by heating for $5 \mathrm{~min}$ at $100^{\circ} \mathrm{C}$ in dissociation buffer $(100 \mu \mathrm{l})$ containing SDS $(2 \%, \mathrm{w} / \mathrm{v})$, glycerol $(10 \%, v / v)$, 2-mercaptoethanol $(5 \%, \mathrm{v} / \mathrm{v})$ and $\mathrm{Tris} / \mathrm{HCl}(125 \mathrm{~mm} ; \mathrm{pH} 6.8)$. The radioactive proteins were analysed by SDS-polyacrylamide gel electrophoresis using acrylamide concentrations of $7 \cdot 5,10$ or $15 \%(w / v)$. A selection of unlabelled proteins were used as standards for $M_{\mathrm{r}}$ determination: glutamate dehydrogenase (cross-linked monomer, 56000-280000), RNA polymerase (165000, 155000, 39000), $\beta$-galactosidase (116000), phosphorylase b (94000), conalbumin (type I, 78000), bovine serum albumin (68000), ovalbumin (43000). lactate dehydrogenase (35000), carbonic anhydrase (29000), trypsin inhibitor protein $(21500)$ and cytochrome $c$ (12500). The gels were stained and then dried for autoradiography.

Peptide mapping. Peptide mapping by limited proteolysis of $\left[{ }^{35} S \mid\right.$ methionine-labelled polypeptides in SDS gels was done according to Cleveland et al. (1977). Bands from stained and autoradiographed gels were treated with 
2 ng Staphylococcus aureus V8 protease (Miles Laboratories) for $15 \mathrm{~min}$ in the stacking gel (5\%) above a second separating gel ( $15 \%$ acrylamide).

Densitometry. Strips of autoradiogram that had been exposed for different periods were scanned at $600 \mathrm{~nm}$. using a Unicam SP1809 scanning densitometer fitted in the sample compartment of a Unicam SP1800 spectrophotometer. The molar ratios of the $a c e E$, aceF and $l p d$ gene products were estimated by dividing the corresponding peak areas by the methionine contents of the respective components. The latter were assumed to be $20 \mathrm{~mol}$ per $100000 \mathrm{~g}$ for E1, $16 \mathrm{~mol}$ per $80000 \mathrm{~g}$ for E2 and $10 \mathrm{~mol}$ per $56000 \mathrm{~g}$ for E3, as reported by Vogel \& Henning (1971. 1973) and Vogel et al. (1971).

Materials. Acrylamide, $N, N^{\prime}$-methylenebisacrylamide and $N, N, N^{\prime}, N^{\prime}$-tetramethylenediamine were obtained from Eastman Chemicals. The sources of other chemicals, enzymes and proteins were: BDH - sodium dodecyl sulphate. Triton X-100 and glutamate dehydrogenase: Boehringer - $\beta$-galactosidase, phosphorylase $b$, lactate dehydrogenase and trypsin inhibitor (soybean): Koch-Light - catalase: Sigma - conalbumin, ovalbumin and carbonic anhydrase; Armour Pharmaceuticals - bovine serum albumin. X-ray film (NS-2T) was from Kodak.

\section{RES ULTS}

The structures of the phages used in this study are summarized in Fig. 1. They contain different segments of an $18.3 \mathrm{~kb}$ region of the $E$. coli chromosome encoding the nadC, aroP, $a c e E, a c e F$ and $l p d$ structural genes. The primary phages, G78N ( $\lambda$ nadC-aroP), G82L ( $\lambda l p d)$ and G83L $(\lambda l p d)$, were originally constructed by cloning $E c o$ RI fragments of bacterial DNA and selected by their ability to transduce and complement specific mutants. Derivative phages, G90 ( $\lambda$ nadC-lpd, from G78N) and G93 ( $\lambda l p d-a c e E$, from G83L), carrying functional aceE, $a c e F$ and $l p d$ genes were subsequently isolated by prophage integration in the nadC or lpd regions and aberrant excision. It should be noted that the bacterial genes of two pairs of phages, G82L and G83L plus G90 and G93, are inserted with opposite polarities. Furthermore, because the orientation of the ace genes relative to the genetic and physical maps is known, it can be deduced that the transcription of the ace operon $(a c e \overrightarrow{E F})$ is polarized rightward in $\mathrm{G} 90\left(\lambda\right.$ nadC-aceEFr-lpd) and leftward in G93 $\left(\lambda l p d-a c e F E^{l}\right)$.

\section{Polypeptides synthesized in a $\lambda$-immune host}

Cultures of the homoimmune host $\mathrm{S} 159$ ( $\lambda \mathrm{imm}^{\lambda}$ ind ${ }^{-}$) were irradiated and labelled after infecting with several transducing phages to analyse phage-directed protein synthesis (Fig. 2). Under these conditions the only bacterial genes that can be expressed are those accompanied by their own promoters. This is because the phage promoters, which could affect the cloned genes, are silent in the immune host.

With G78N ( $\lambda$ nadC) only one specifically-labelled polypeptide, $M_{\mathrm{r}} 36500$, was detected. The same labelled product was also formed with $\mathrm{G} 90(\lambda$ nadC-lpd) but not with $\mathrm{G} 83 \mathrm{~L}(\lambda l p d)$ or G93 ( $\lambda l p d-a c e E$ ). This polypeptide, $M_{\mathrm{r}} 36500$, could be the $n a d C$ or aroP gene product.

The lpd transducing phage, G83L, directed the synthesis of only one specific product and this was readily identifiable, by its size $\left(M_{\mathrm{r}}\right.$ 56500), as lipoamide dehydrogenase (Fig. 2). This confirms that the lpd gene has its own promoter and can be expressed independently of the ace operon. With the phages containing functional ace and $l p d$ genes, G90 and G93, the labelled polypeptides corresponded to the three components of the pyruvate dehydrogenase complex: $\mathrm{E} 1,100000(a c e E)$; E2, 80000-83000 (aceF); and E3, 56500 (lpd). The E2 region was noticeably diffuse, occupying an $M_{\mathrm{r}}$ range of 80000 to 83000 . This is probably due to heterogeneity of the E2 component. The presence of at least two polypeptides migrating at different rates was an invariant feature of this investigation. Quantitative densitometry of the autoradiograms (Fig. 3) gave reproducible values for the relative incorporation of methionine into the pyruvate dehydrogenase complex components during the $20 \mathrm{~min}$ period of synthesis. The ratios (E1 :E2:E3) were $1 \cdot 13: 1.00: 0 \cdot 87$ (G93) and 1.18:1.00:1.44 (G90). By making assumptions about the size and composition of the components (see Methods) these correspond to the molar ratios (E1:E2:E3) or $0.94: 1 \cdot 00: 1.40$ (G93) and $0.94: 1 \cdot 00: 2 \cdot 30$ 


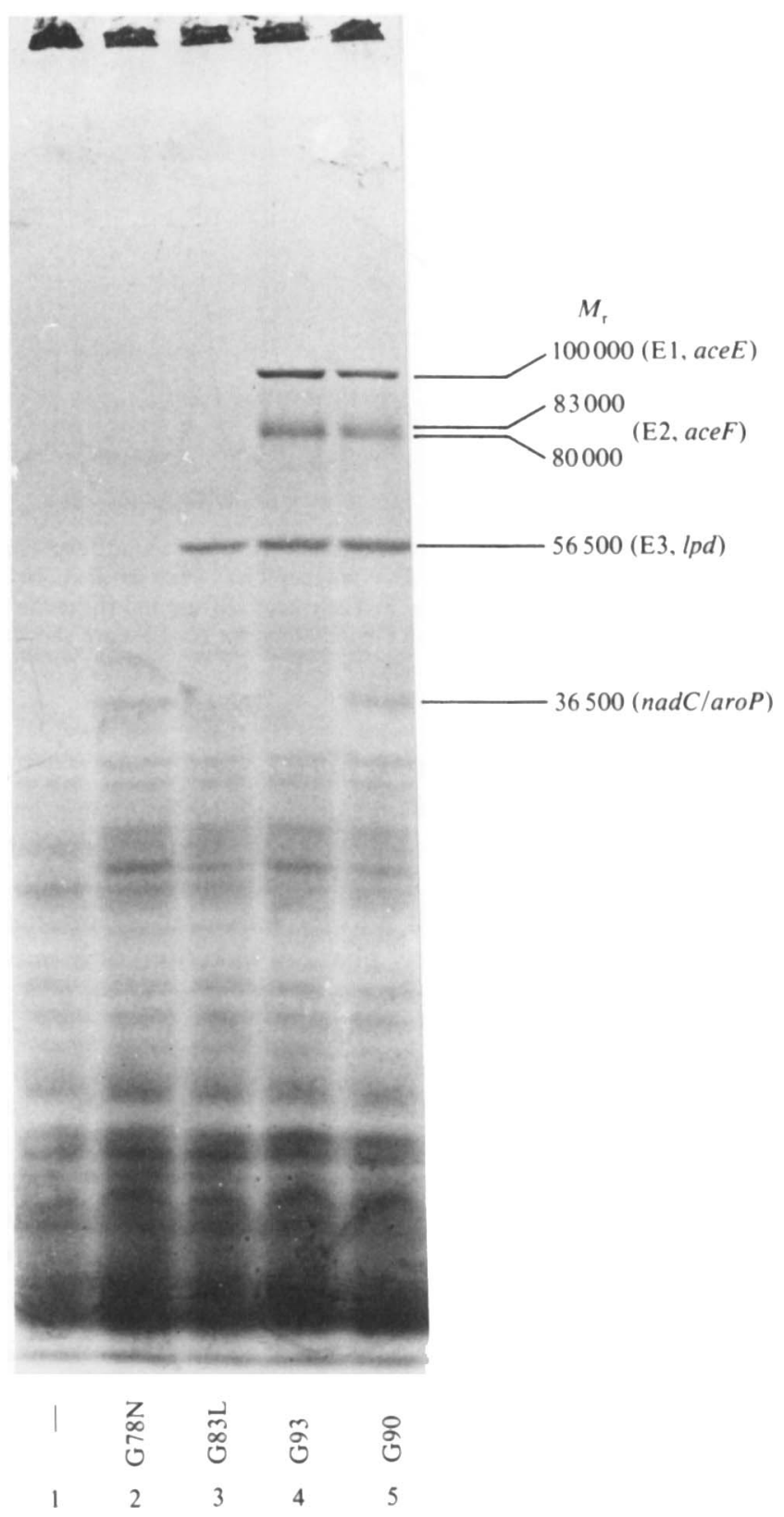

Fig. 2. Autoradiogram of ${ }^{35} \mathrm{~S}$-labelled polypeptides synthesized in a u.v.-irradiated $\lambda$-immune host, $S 159$ $\left(\lambda \mathrm{imm}^{\lambda}\right.$ ind), after infection with several transducing phages and fractionation in SDS-polyacrylamide gel (10\%). Labelling was for $20 \mathrm{~min}$. The sizes of the products specifically directed by cloned genes were estimated by comparison with unlabelled standards. The tracks correspond to the following phages: 1 , no phage (uninfected bacteria); 2, G78N ( $\lambda$ nadC); 3, G83L ( $\lambda l p d) ; 4, \mathrm{G} 93$ ( $\lambda l p d-a c e E) ; 5, \mathrm{G} 90$ $(\lambda$ nadC-lpd).

(G90). The same ratios were observed with other transducing phages of similar types. The reason for the different level of incorporation into the E3 component is unknown, but it should be remembered that the bacterial genes are cloned with opposite polarities in these phages. 


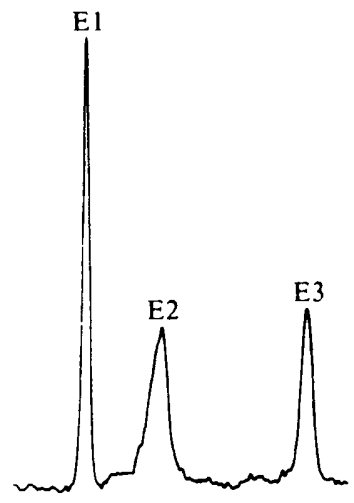

(a) G93 (גlpd-aceE)

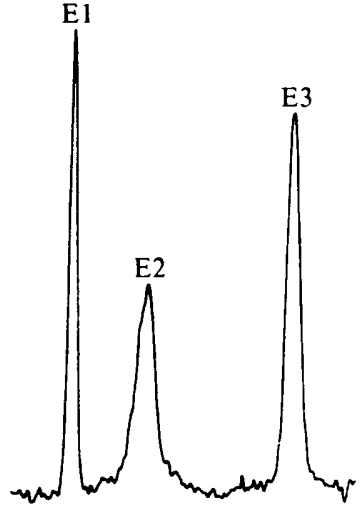

(b) $\mathrm{G} 90($ inadC-lpd)

Fig. 3. Densitometer traces of autoradiograms of ${ }^{35} \mathrm{~S}$-labelled polypeptides expressed from pyruvate dehydrogenase complex transducing phages. The polypeptides were labelled for 20 min following infection of the $\lambda$-immune host described in Fig. 2. The traces correspond to tracks $4(a)$ and $5(b)$ in Fig. 2: only the regions containing components of the multienzyme complex are shown.

\section{Polypeptides synthesized in a $\lambda$-sensitive host}

Expression of the cloned genes was also examined by infecting and pulse-labelling a u.v.-irradiated $\lambda$-sensitive host (S159). Under these conditions the products of the cloned genes are seen against a background of $\lambda$-specified proteins. Also, depending on the orientation of the cloned genes, expression may be enhanced or retarded by the powerful leftward phage promoter $P_{L}$ (during the early phase of infection) or it may be affected by the late rightward promoter $P_{R}^{\prime}$ (during the late phase of infection, when transcription from $P_{L}$ is repressed). Thus, genes with a leftward ( $l$ ) orientation benefit from the early effects of $\mathrm{P}_{\mathrm{L}}$ and genes with the opposite, rightward ( $r$ ) orientation are inhibited in the early phase but can later be expressed at their inherent rate or at a rate enhanced by rightward transcription originating at $\mathbf{P}_{\mathrm{R}}^{\prime}$. In addition, genes without promoters can be expressed from the phage promoters, and hybrid genes, generated by fusion of phage and bacterial DNA at restriction targets, can be expressed from phage or bacterial promoters, and these are subject to the same temporal constraints imposed by their orientations. The labelling periods used were 3-13 min (early) and 25-35 min (late) after infection.

Orientation of lpd and ace gene expression. Two $\lambda l p d$ phages were examined (G82L and G83L) with $\lambda \operatorname{nadC}(\mathrm{G} 78 \mathrm{~N})$ as a control (Fig. 4). During the early period, incorporation into the lipoamide dehydrogenase polypeptide $\left(M_{\mathrm{r}}\right.$ 56500) was much higher with G83L than G82L, but during the late period there was very little difference. The E3 component is better resolved from a phage protein of about the same size in the $7.5 \%$ gel (Fig. $4 b$ ). Because expression of the lpd gene is enhanced in G83L, this phage can be designated $\lambda l p d^{l}$ to denote the leftward orientation of the lpd gene. By contrast, the lpd gene of G82L must have the rightward orientation, because its expression appears to be inhibited until transcription from $P_{L}$ is shut off by the $\lambda$ cro function. Phage G82L may thus be designated $\lambda l p d r$. Another phage, G105 ( $\lambda l p d)$, was also designated $\lambda l p d^{l}$ because, as with G83L, labelling of the lpd product was high in both the early and late periods. This is consistent with the physical structures of G105 and G83L, which show the bacterial DNA cloned with the same polarity (Fig. 1).

Polypeptides corresponding to the dihydrolipoamide acetyltransferase component (E2, $a c e F$ ) were also labelled with phages G82L (late) and G83L (early and late). Their molecular weights, in decreasing order of intensity, were 80000 (major), 83000 and 86000 (minor). The $M_{\mathrm{r}} 80000$ and 83000 components sometimes appeared in equivalent amounts (Fig. $4 \mathrm{a}$ ); the 
minor component $\left(M_{\mathrm{r}} 86000\right)$ was better resolved in $7.5 \%$ gels (Fig. $\left.4 b\right)$. The reason for this heterogeneity is not known. Some lessening of the relative amounts of the larger components $\left(M_{\mathrm{r}} 83000\right.$ and 86000) was observed following storage of the samples for 2 weeks at $-20^{\circ} \mathrm{C}$ before analysis.

Phage G83L specifically directed the labelling of a prominent polypeptide of $M_{\mathrm{r}} 90000$ (Fig. 4). This was shown to contain part of the aceE gene product (E1) by peptide mapping studies with the isolated band. Parallel digestion of comparable samples of this polypeptide and the E1 component $\left(M_{\mathrm{r}} 100000\right)$ with $S$. aureus V8 protease produced 19 and 23 labelled fragments, respectively. Of these, 12 were common to both, the others being specific to the $M_{\mathrm{r}}$ 90000 polypeptide (7) and E1 (11). Thus it would appear that the $M_{\mathrm{r}} 90000$ polypeptide is the product of fusion between a phage gene and the $a c e E$ gene. The failure to detect this product in the late period of labelling with G82L, in which the EcoRI fragment is inverted, is consistent with this conclusion. The results indicate that the EcoRI fragment $(8.2 \mathrm{~kb})$ cloned in G82L not only contains a functional lpd gene but also an intact ace $F$ gene and a significant part of the aceE gene. However, these ace sequences must be separated from the ace promoter because their products were not observed in an immune host, where the $\lambda$ promoters are inactive, but they were expressed when linked to active $\lambda$ promoters. These results are consistent with the known polarity of the ace operon and the conclusions of Guest \& Stephens (1980) that the critical EcoRI target is in the aceE gene and that the corresponding $\lambda l p d$ phages contain all of the $a c e F$ gene but only part of the aceE gene. No comparable fusion products were detected with the $\lambda$ lpd phage, G105. This contains the $5.4 \mathrm{~kb} H$ indIII plus $E c o R I$ fragment but should encode part of the $a c e F$ gene as well as the lpd gene.

Other polypeptides observed when the E2 component was strongly labelled had molecular weights of $72000,69000,43000$ and 42000 (Fig. 4 b). These could be E2 degradation products because this component is known to be unstable. It generates a series of comparably sized intermediates and eventually active sub-components of about 39000 to 35000 (Hale \& Perham, 1979; Gebhardt et al., 1978). If formed, the ultimate product may be obscured by the prominent $\lambda E$ gene product at 37000 (Fig. 4).

In addition to several identifiable phage proteins, two other peptides, $M_{\mathrm{r}} 78000(\mathrm{G} 82 \mathrm{~L})$ and 120000 (G83L), were synthesized as early- and late-labelled products (Fig. 4). These were probably generated by fusion between phage and bacterial genes and their orientationdependent formation supports this conclusion.

Comparable early- and late-labelling experiments with phages carrying functional ace-lpd regions, G93 ( $\lambda l p d-a c e E)$ and G90 $(\lambda$ nadC-lpd), are shown in Fig. 5. These confirmed that the ace and lpd genes are both transcribed with the same polarity, their expression being leftward in G93 ( $\left.\lambda l p d^{l}-a c e^{l}\right)$ and rightward in G90 ( $\lambda$ nadC-acer-lpdr). The aceE and lpd products were identifiable as polypeptides of $M_{\mathrm{r}} 100000$ and 56500 , respectively. However, with these phages the heterogeneity in the acetyltransferase (E2) region was even greater. There appeared to be a family of polypeptides of increasing size $\left(M_{\mathrm{r}} 80000,83000,86000\right.$, 89000 and 91000), but decreasing intensity. These were quite specific and distinct from a phage protein that migrates to this region, the late $\lambda H$ gene product $\left(M_{\mathrm{r}} 88000\right)$. Also with G93 ( $\left.\lambda l p d^{\prime}-a c e\right)$, a very large component $\left(M_{\mathrm{r}} 190000\right)$ was invariably present but its exact nature was difficult to define (see below). The postulated E2 degradation products were also visible.

Polypeptide products of the nadC-aroP region. Studies with the $\lambda$-immune host identified a polypeptide, $M_{\mathrm{r}} 36500$, which was specifically expressed from phages carrying the nadC-aroP region (G78N and its derivative, G90). A product of the same size, formed at an enhanced rate in the late phase of $\lambda$ nadC infection, was detected with the $\lambda$-sensitive host. This suggests that the corresponding gene is transcribed with rightward orientation. However, this could not be correlated with nadC or aroP, the only genes known to be located in the $10.1 \mathrm{~kb}$ EcoRI fragment. Other specific polypeptides, probably derived by rightward (29500 and 
(a) $10 \%$ acrylamide

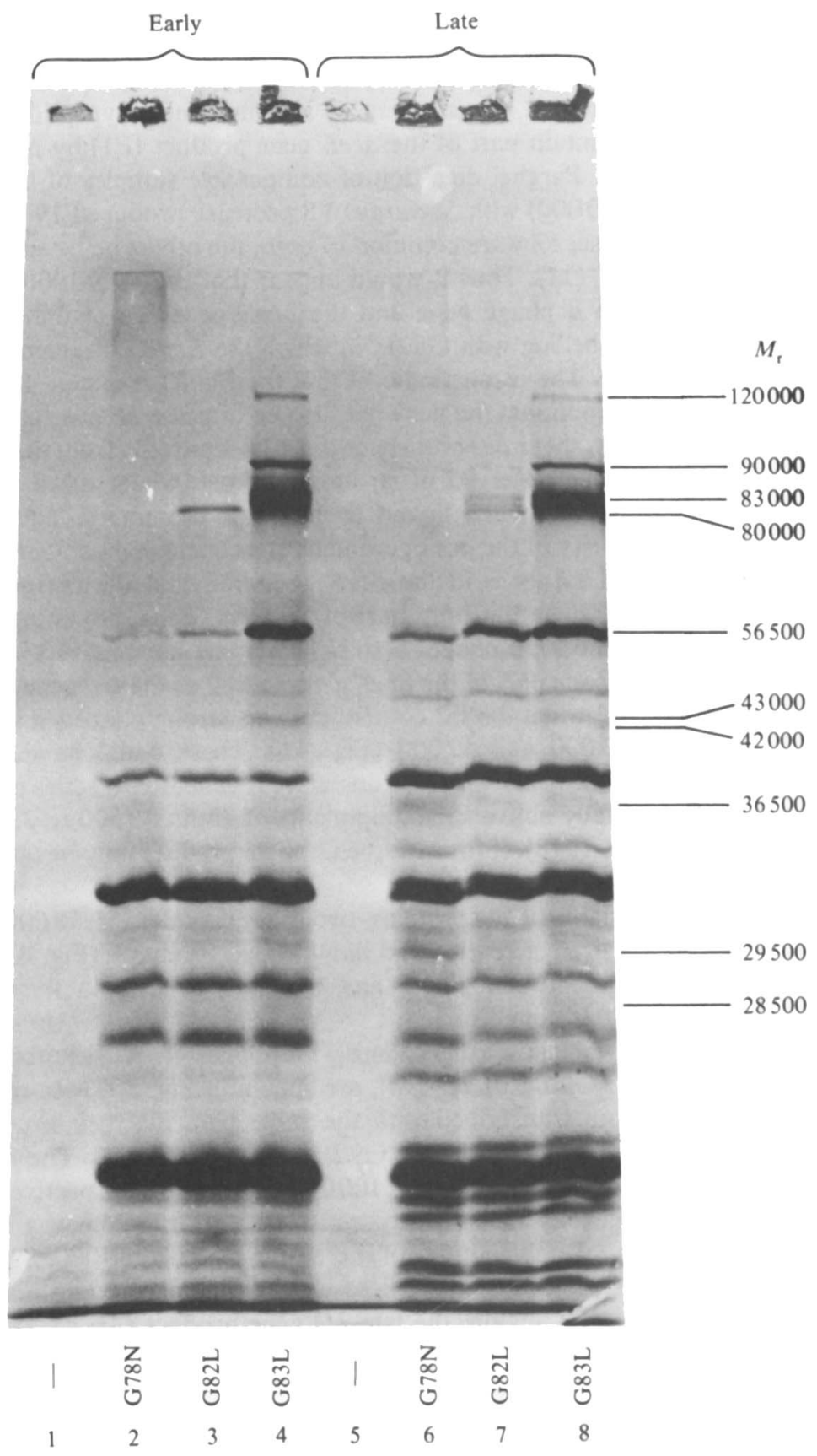

Fig. 4. Autoradiograms of the ${ }^{35} \mathrm{~S}$-labelled polypeptides formed after infection of a u.v.-irradiated $\lambda$-sensitive host (S159) with $\lambda$ nadC and $\lambda l p d$ phages. Labelling was for $10 \mathrm{~min}$ in either the early (3-13 min. tracks $1-4)$ or late (25-35 min. tracks 5-8) periods after infection with the following phages: 1 and 5, no phage; 2 and $6, \mathrm{G} 78 \mathrm{~N}(\lambda \operatorname{nadC}) ; 3$ and 7, G82L $\left(\lambda l p d^{r}\right) ; 4$ and 8, G83L $\left(\lambda l p d^{\prime}\right)$. Samples of the labelled cultures were analysed by electrophoresis in SDS gels containing $10 \%(a)$ and $7.5 \%(b)$ acrylamide. A sample of the material shown in Fig. $3(a)$ provided radioactive markers for the E1, E2 and E3 components (track 9). 
(b) $7.5 \%$ acrylamide

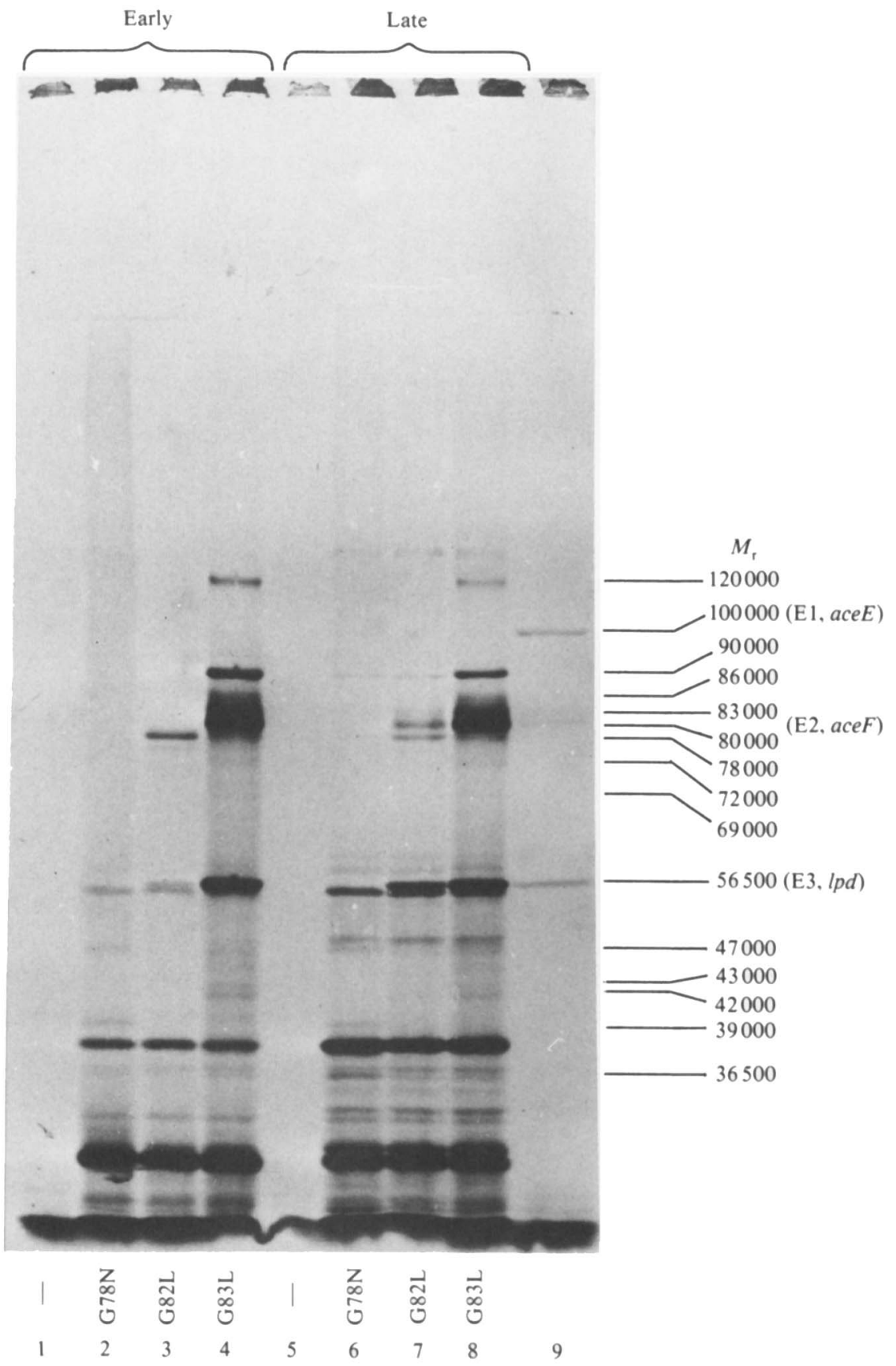




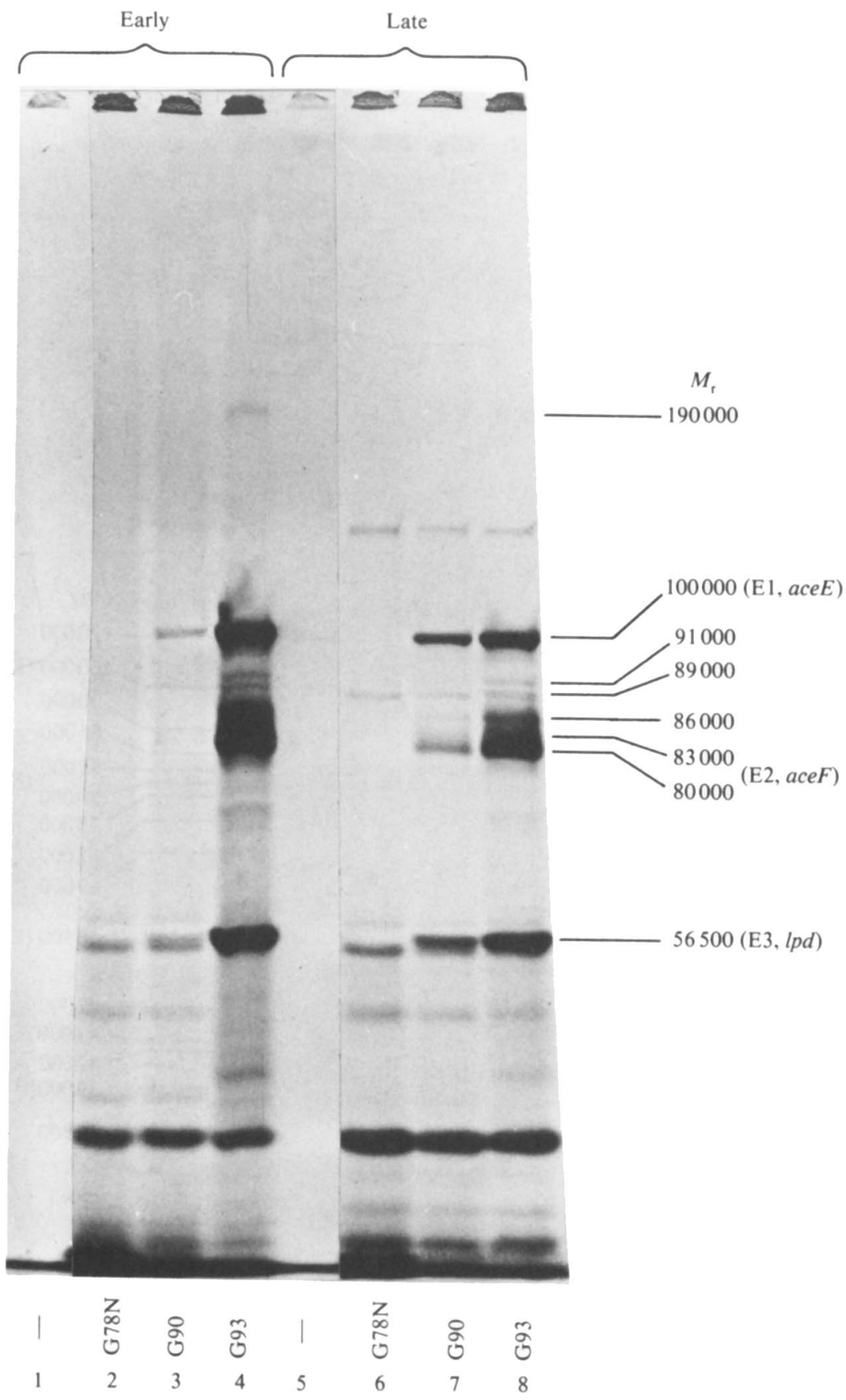

Fig. 5. Autoradiogram of ${ }^{35} \mathrm{~S}$-labelled polypeptides produced after infection of a u.v.-irradiated $\lambda$-sensitive host (S159) with transducing phages containing the three pyruvate dehydrogenase complex genes ( $\lambda$ nadC-lpd and $\lambda l p d-a c e E$ ). The early- and late-labelling procedures were as described in Fig. 4 and the SDS gel contained $7.5 \%$ acrylamide. The tracks correspond to the following phages: 1 and 5 , no phage (uninfected bacteria): 2 and $6, G 78 \mathrm{~N}$ ( $\lambda$ nadC) control for phage gene expression: 3 and 7 ,

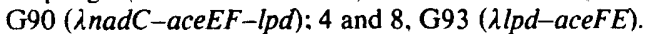




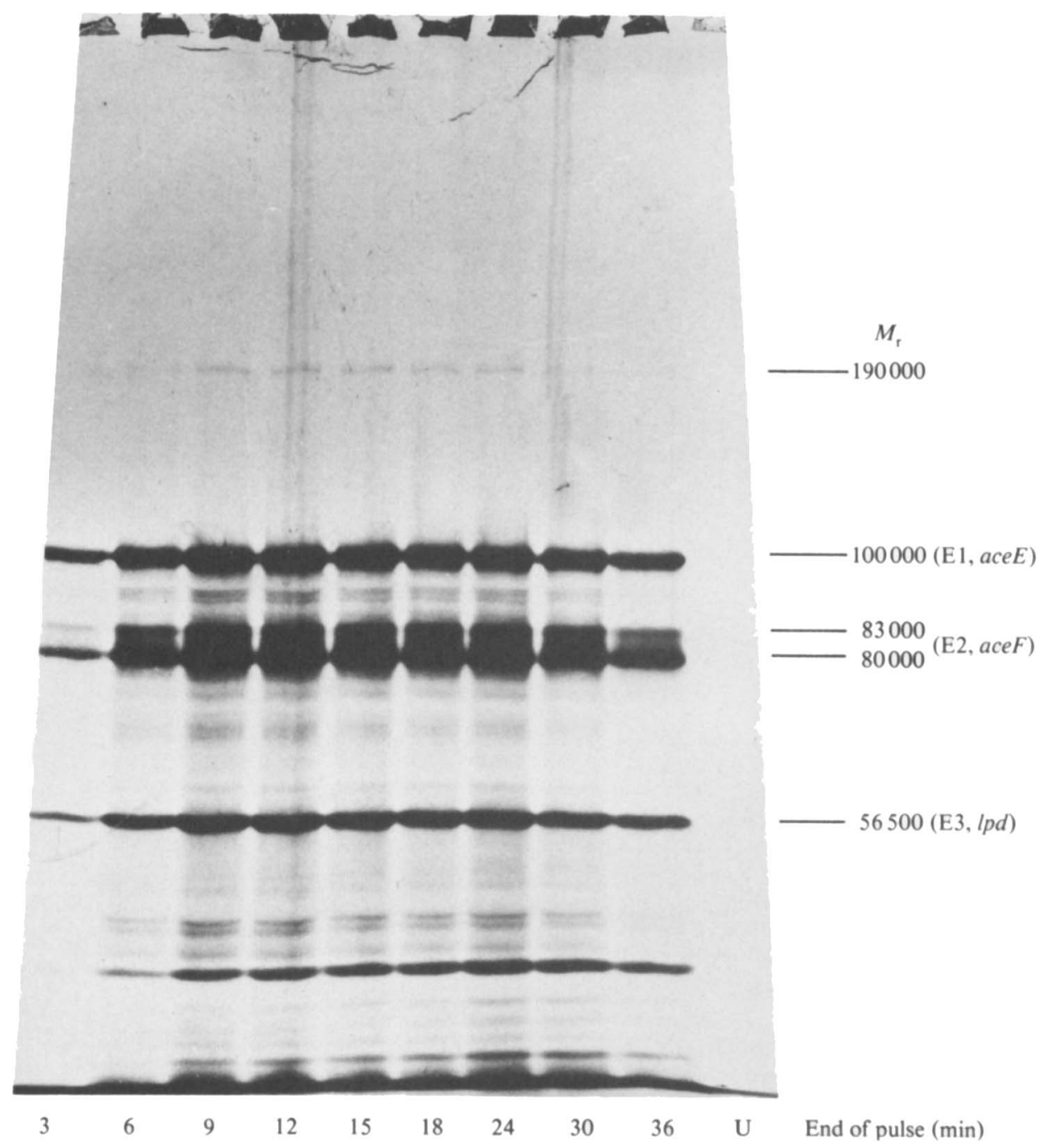

Fig. 6. Autoradiographic analysis of ${ }^{35} \mathrm{~S}$-labelled polypeptides produced in a u.v.-irradiated $\lambda$-sensitive host (S159) after infection with G93 $\left(\lambda l p d^{d}-a c e^{\prime}\right)$ and exposure to ${ }^{35} S \mid$ methionine for 3 or 6 min periods before electrophoresis through a $\mathbf{7 . 5} \%$ polyacrylamide gel containing SDS. The numbers on each track indicate the time ( $\mathrm{min}$ ) of termination of each pulse $(3 \mathrm{~min}$ for the 3 to $18 \mathrm{~min}$ samples and $6 \mathrm{~min}$ for the 24 to 36 min samples). The track marked $U$ represents uninfected bacteria labelled in the 30 to $36 \mathrm{~min}$ period.

28500 ) and leftward (47000 and 39000 ) transcription, were also detected with the $\lambda$-sensitive host but their origin is unknown.

Progress of ace-lpd gene expression. Temporal changes in expression of the aceE, aceF and $l p d$ genes were investigated by infecting the $\lambda$-sensitive host with G93 $\left(\lambda l p d^{l}-a c e^{\lambda}\right)$ and labelling with pulses of 3 or $6 \mathrm{~min}$ over a period of $36 \mathrm{~min}$ (Fig. 6). All the components were detectable from the earliest time and their rates of synthesis increased steadily for 9 min before levelling off and then declining after 18-24 min. The molar ratios were approximately $1: 1: 1$ $(\mathrm{E} 1: \mathrm{E} 2: \mathrm{E} 3)$ in the early stages but after $9 \mathrm{~min}$ there appeared to be less E1 and more E3 relative to the sum of the main E2 components $\left(M_{\mathrm{r}} 80000\right.$ and 83000$)$ thus: $0 \cdot 5: 1 \cdot 0: 1 \cdot 3$ (E1 :E2:E3). It was also clear that the components of the multienzyme complexes accounted 


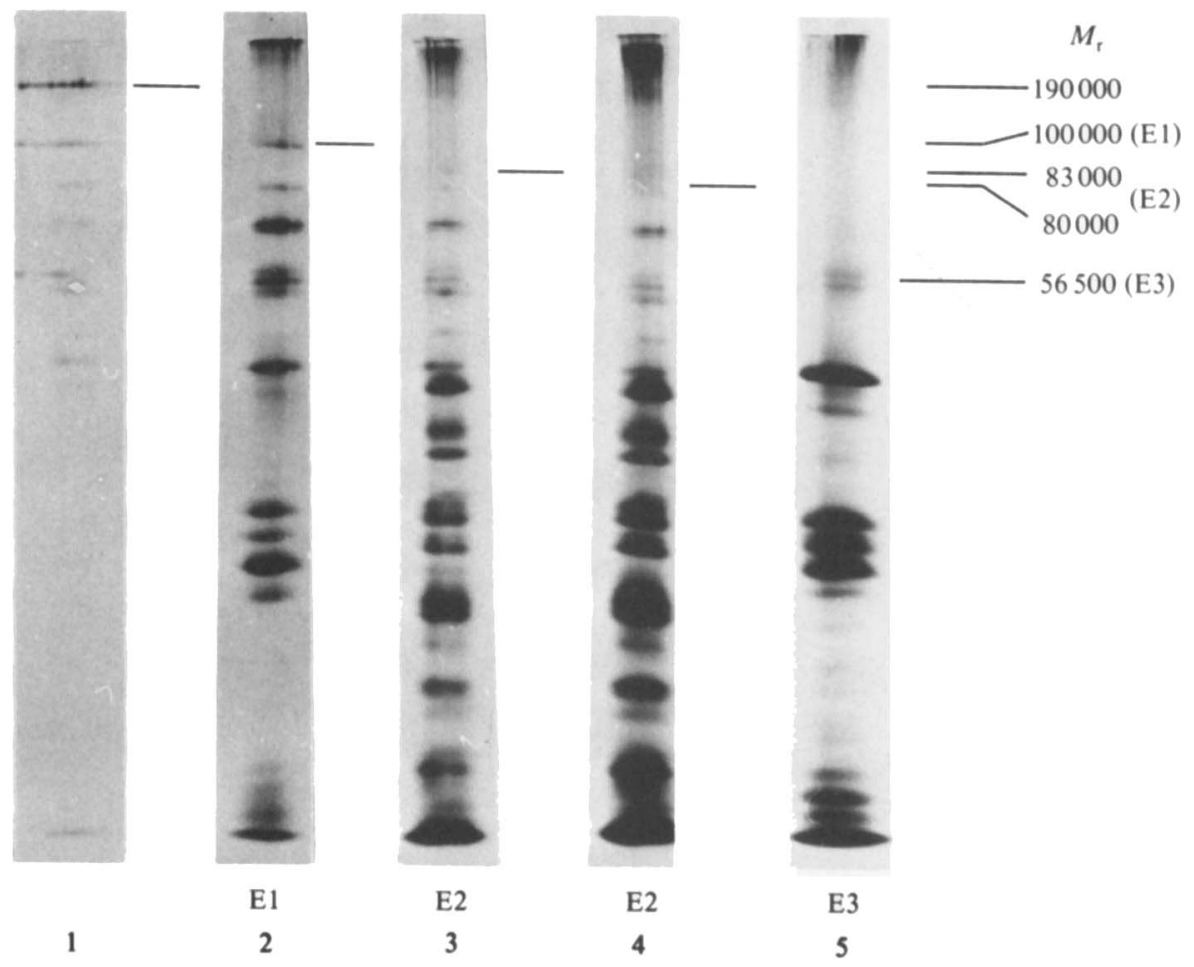

Fig. 7. Peptide mapping of the large polypeptide $\left(M_{\mathrm{r}}\right.$ 190000) and other components of the pyruvate dehydrogenase complex. The products of digestion of the ${ }^{35} \mathrm{~S}$-labelled polypeptides were separated by electrophoresis in a $15 \%$ polyacrylamide gel containing SDS and located autoradiographically. The tracks correspond to polypeptides of the following sizes: $1, M_{\mathrm{r}} 190000 ; 2,100000(\mathrm{E} 1) ; 3,83000$ (E2 minor component); 4, 80000 (E2 major component); 5, 56500 (E3). The positions of the untreated polypeptides in the same gel are indicated by the horizontal bars.

for the greater part of the newly synthesized protein. These findings were confirmed by parallel cumulative labelling studies, which showed a continuous increase in the amounts of the three gene products up to $30 \mathrm{~min}$ after infection. In addition to the heterogeneous E2 region and its presumptive degradation products, a very large polypeptide $\left(M_{\mathrm{r}} 190000\right)$ was observed. From its size this could conceivably represent an E1-E2 fusion product translated from the aceE-aceF messenger. This possibility was explored by subjecting the excised band to limited proteolysis with $S$. aureus V8 protease followed by electrophoresis and autoradiography (Fig. 7). Well-separated samples of the other major components were analysed by the same method. The major components generated characteristic proteolytic fragmentation patterns which were identical to those obtained previously with stained preparations of the E1, E2 and E3 components of the $E$. coli pyruvate dehydrogenase complex (Jeyaseelan et al., 1980; Jeyaseelan, 1980). The two main forms of the E2 component $\left(M_{\mathrm{r}} 80000\right.$ and 83000$)$ were indistinguishable, indicating that they have a common origin. The very large polypeptide $\left(M_{\mathrm{r}} 190000\right)$ seemed to be rather resistant to proteolysis and the few fragments which were detected resembled the products of E1 digestion but not E2. This does not totally exclude the presence of E2 sequences but it does indicate that the $M_{\mathrm{r}} 190000$ material contains E1 sequences. This material could arise by aggregation of the polypeptides of a dimer followed by incomplete resolution in the denaturing conditions used for preparing samples for SDS-polyacrylamide gel electrophoresis. In this connection, even larger molecules $\left(M_{r} 270000\right.$ and 310000$)$ were occasionally observed. These could represent higher aggregates of El components with or without other components of the 
multienzyme complex, detectable due to the high sensitivity of the autoradiographic method. No precursor-product relationships between any of the E2 components or for the very large polypeptides were apparent from the labelling studies.

\section{DISCUSSION}

The existence of $\lambda$ transducing phages containing the pyruvate dehydrogenase complex genes provided an excellent opportunity for studying ace and lpd gene expression over short periods of time in vivo. Using appropriate phages, the $a c e E$, aceF and $l p d$ gene products were identifiable as labelled polypeptides. Their synthesis in a $\lambda$-immune host indicated that these genes are accompanied by their own promoters, one for the two ace genes and another for the lpd gene. This confirmed earlier observations on the expression of the ace gene (Henning et al., 1966) and the independence of the lpd gene (Langley \& Guest, 1978; Guest \& Stephens, 1980). By analysing the early- and late-labelled polypeptides synthesized after infecting a non-lysogenic host, the polarities of expression of the cloned genes could be defined relative to the phage promoter. Thus the ace and lpd genes cloned in the phages depicted in Fig. 1 were found to be expressed with rightward (G90 and G82L) and leftward (G83L, G93 and G105) orientation. This showed for the first time that the lpd gene is expressed with the same polarity as the ace operon (Fig. 8): clockwise with respect to the $E$. coli linkage map. Because they have the same polarity it is conceivable that transcription originating at $\mathrm{P}_{\text {ace }}$ could continue through the $l p d$ gene without termination near $\mathrm{P}_{l p d}$. If so, independent transcription from $\mathrm{P}_{l p d}$ may normally be important only for the synthesis of the 2-oxoglutarate dehydrogenase complex. However, this function would be essential when the ace genes are repressed. It is anticipated that the nature of the transcripts generated from this region will emerge during further studies of the expression of the cloned genes.

The results confirmed and extended previous correlations of the restriction and genetic maps (Fig. 8). The central EcoRI target is clearly situated in the aceE gene because the original $\lambda l p d$ phages (G83L), containing the $8.2 \mathrm{~kb} E c o R I$ fragment, expressed an intact ace $F$ product (the acetyltransferase component, E2) and a considerable portion of the aceE product, as well as lipoamide dehydrogenase, when coupled to a $\lambda$ promoter. Furthermore, the $5.4 \mathrm{~kb}$ sub-fragment of phage G105 encoded a functional lpd gene. The $\lambda$ nadC phages expressed only one detectable bacterial product $\left(M_{\mathrm{r}} 36500\right)$. This could be quinolinate phosphoribosyltransferase or the general aromatic amino acid permease, the only functions known to be cloned in these phages. The segment of DNA represented by the two EcoRI fragments $(18.3 \mathrm{~kb})$ encodes only five known genes and the four detectable products have an approximate coding requirement of only $8.5 \mathrm{~kb}$. This considerable excess of coding capacity indicates that there are several undetected genes in this region or that it contains a significant proportion of unexpressed DNA. It is known that the entire nadC-lpd region can be deleted without loss of any essential functions other than the five genes already mentioned (Langley \& Guest, 1978).

Quantitative densitometry of the labelled pyruvate dehydrogenase complex components, expressed from the $\lambda$-cloned genes in a lysogenic host, clearly indicates that the E1 and E2 components are synthesized in almost equal molar proportions whereas there is a molar excess of E3 components. Under these conditions the genes are expressed solely from their own promoters. Consequently, there would not seem to be any inherent modulation of E1 and E2 synthesis encoded in the ace operon. Complexed and uncomplexed components were not resolved in the present analysis but the results are consistent with the early $1: 1$ (E1:E2) stoichiometries reported for the isolated pyruvate dehydrogenase complex (Eley et al., 1972) and the core complex (Vogel et al., 1972). It would appear that the isolated complexes reported to contain a molar excess of $\mathrm{E} 1$ components could be due to post-translational events such as differential assembly or stability of the components or to some translational 


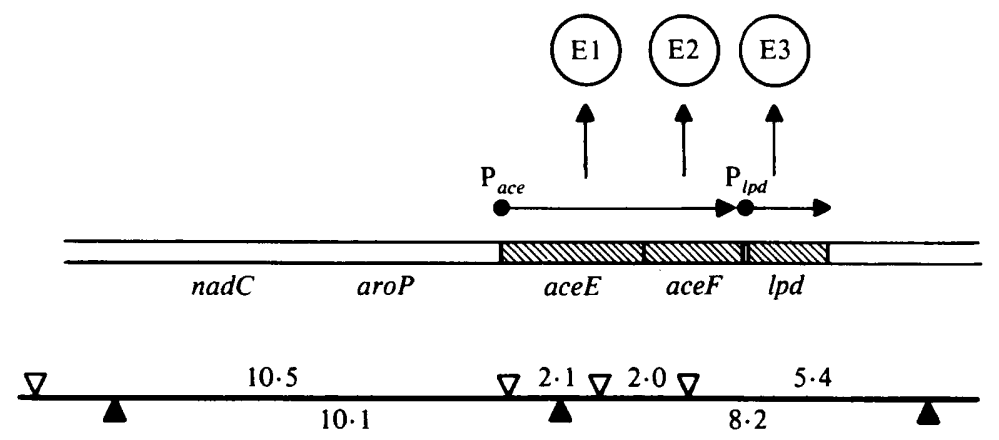

Fig. 8. Diagram showing the transcriptional polarities of the pyruvate dehydrogenase complex genes relative to the genetic and physical maps of the nadC-lpd region $(2.5$ to $2.8 \mathrm{~min})$. The diagram is drawn to scale: the positions of the ace and $l p d$ genes relative to the restriction map are based on previous work (Guest \& Stephens. 1980), present results and unpublished observations. The sizes of the ace and lpd genes are derived from the molecular weights of their products, and the corresponding promoters are denoted $\mathrm{P}_{a c e}$ and $\mathrm{P}_{l p d}$. The restriction targets for HindIII $(\nabla)$ and EcoRI $(\Delta)$ are indicated and the fragment sizes are in kilobase pairs $(\mathrm{kb})$.

modulation or attenuation mechanism that could be geared to the physiological state of the organism.

It is not possible to relate the labelling of the E3 component to complex structures because two complexes are involved. However, the 1.4-2.3-fold excess of E3 components relative to the ace gene products confirms the presence of an lpd promoter that functions independently, at least when providing components for assembly into the 2-oxoglutarate dehydrogenase complex. Transcription of the ace and $l p d$ genes in a non-lysogenic host can originate from both bacterial and phage promoters and the labelling patterns in the early phase of infection exhibited a $1: 1: 1$ (E1:E2:E3) stoichiometry. Most of the newly synthesized protein was represented by the three components and it would appear that the results reflect the overriding influence of the powerful leftward phage promoter $\mathrm{P}_{\mathrm{L}}$. At a later stage, deviations from this stoichiometry were observed but these could be due to the temporal changes in phage-directed transcription causing differential effects on the progress of ace and lpd gene expression.

The ability to label the gene products with a relatively high specific radioactivity and then analyse the polypeptides of the whole cells within minutes of their synthesis led to the discovery of considerable heterogeneity amongst the polypeptides designated ace $F$ products, as well as a very large polypeptide $\left(M_{\mathrm{r}} 190000\right)$. The E2 component was almost invariably represented by two bands, $M_{\mathrm{r}} 80000$ and 83000 , and in some cases these were accompanied by a family of minor components of approximately $M_{\mathrm{r}} 86000,89000$ and 91000 . The double-banded nature of the E2 component has been observed with preparations of the isolated $E$. coli complex (unpublished observations) and with the corresponding complex of Pseudomonas aeruginosa (Jeyaseelan et al.. 1980). If it is not an artefact it may have some structural significance with respect to the assembly of the complex. The extended heterogeneity could also correspond to stages in the processing of a large primary gene product into the smaller polypeptides of the complex. This component is noted for its instability and it may be significant that using a faster purification, Gebhardt et al. (1978) have indicated that the native acetyltransferase is larger $\left(M_{\mathrm{r}} 89000\right)$ than is generally reported but decays via a fragment of $M_{\mathrm{r}} 82000$. Unfortunately, no precursor-product relationships could be detected for the labelled polypeptides. The same was true for the $M_{\mathrm{r}} 190000$ polypeptide, which contained E1 sequences: its nature and origin remain obscure.

We are indebted to Dr N. E. Murray for helpful discussions and advice. This work was supported by the Science Research Council (GR/B35543) and K.J. acknowledges a postgraduate studentship from the Commonwealth Commission in the United Kingdom. 


\section{REFERENCES}

Bleile, D. M., Munk, P., Oliver, R. M. \& Reed, L. J. (1979). Subunit structure of dihydrolipoyltransacetylase component of pyruvate dehydrogenase complex of Escherichia coli. Proceedings of the National Academy of Sciences of the United States of A merica 76, 4385-4389.

Cleveland, D. W., Fischer, S. G., Kirschner, M. W. \& Laemml, U. K. (1977). Peptide mapping by limited proteolysis in sodium dodecyl sulphate and analysis by gel electrophoresis. Journal of Biological Chemistry 252, 1102-1106.

Creaghan, I. T. \& Guest, J. R. (1972). Amber mutants of the $\alpha$-ketoglutarate dehydrogenase gene of Escherichia coli K12. Journal of General Microbiology 71, 207-220.

Danson, M. J., Hale, G., Johnson, P., Perham, R. N., Smith, J. \& SpragG, P. (1979). Molecular weight and symmetry of the pyruvate dehydrogenase multienzyme complex of Escherichia coli.Journal of Molecular Biology 129, 603-617.

Eley, M. H., Namihara, G., Hamilton, L., MunK, P. \& REED, L. J. (1972). $\alpha$-Keto acid dehydrogenase complexes XVIII. Subunit composition of the Escherichia coli pyruvate dehydrogenase complex. Archives of Biochemistry and Biophysics 152, 655-669.

Gebhardt, T., Mecke, D. \& Bisswanger, H. (1978). Dihydrolipoamide transacetylase from Escherichia coli: evidence for internal gene duplication. Biochemical and Biophysical Research Communications 84, 508-514.

GuEST, J. R. (1978). Aspects of the molecular biology of lipoamide dehydrogenase. Advances in Neurology 21, 219-244.

Guest, J. R. \& Stephens, P. E. (1980). Molecular cloning of the pyruvate dehydrogenase complex genes of Escherichia coli. Journal of General Microbiology 121, 277-292.

Hale, G. \& Perham, R. N. (1979). Limited proteolysis of the pyruvate dehydrogenase complex of Escherichia coli. European Journal of Biochemistry 94, 119-126.

HenNing, U., Dennert, G., Hertel, R. \& Shipp, W.S. (1966). Translation of the structural gene of the $E$. coli pyruvate dehydrogenase complex. Cold Spring Harbor Symposia on Quantitative Biology 31, 227-234.
JeyASEelan, K. (1980). Biochemical and genetic studies of bacterial pyruvate dehydrogenase complexes. Ph.D. thesis, Sheffield University, U.K.

Jeyaseelan, K., Guest, J. R. \& Visser, J. (1980). The pyruvate dehydrogenase complex of Pseudomonas aeruginosa PAO: purification, properties and characterization of mutants. Journal of General Microbiology 120, 393-402.

LANGley, D. \& Guest, J. R. (1978). Biochemical genetics of the $\alpha$-keto acid dehydrogenase complexes of Escherichia coli K12: genetic characterization and regulatory properties of deletion mutants. Journal of General Microbiology 106, 103-117.

MilleR, J. H. (1972). Experiments in Molecular Genetics. New York: Cold Spring Harbor Laboratory.

Murray, N. E., Brammar, W. J. \& Murray, K. (1977). Lambdoid phages that simplify the recovery of in vitro recombinants. Molecular and General Genetics 150, 53-61.

Schmit, B. \& Cohen, R. (1980). The structure of the Escherichia coli pyruvate dehydrogenase complex is probably not unique. Biochemical and Biophysical Research Communications 93, 709-712.

VOGEL, O. (1977). Redetermination of the molecular weights of the components of the pyruvate dehydrogenase complex from $E$. coli $\mathrm{K} 12$. Biochemical and Biophysical Research Communications 74, 12351241.

Vogel, O. \& Henning, U. (1971). Pyruvate dehydrogenase component subunit structure of the Escherichia coli K12 pyruvate dehydrogenase complex. European Journal of Biochemistry 18, 103-115.

Vogel, O. \& Henning, U. (1973). The subunit structure of the Escherichia coli K12 pyruvate dehydrogenase complex. European Journal of Biochemistry 35, 307-310.

Vogel, O., Beikirch, H., Muller, H. \& Henning, U. (1971). The subunit structure of the Escherichia coli K12 pyruvate dehydrogenase complex: the dihydrolipoamide transacetylase component. European Journal of Biochemistry 20, 169-178.

Vogel, O., Hoehn, B. \& Henning, U. (1972). Molecular structure of the pyruvate dehydrogenase complex from Escherichia coli K-12. Proceedings of the National Academy of Sciences of the United States of America 69, 1615-1619. 